\title{
MODELO DE GESTIÓN ASISTENCIAL BASADO EN EL PRINCIPIO DE ACCESIBILIDAD TOTAL
}

\section{HEALTHCARE ADMINISTRATION DESIGN ESTABLISHED ON A TOTAL-ACCESS BASIS}

\author{
GEGÚNDEZ-FERNÁNDEZ JA ${ }^{1}$
}

Hoy en día la valoración de la satisfacción del paciente es el parámetro más importante en la calidad asistencial, lo que modifica la visión de la prestación sanitaria que hasta ahora se tenía. Por esta razón, lo servicios sanitarios deben orientarse principalmente hacia los ciudadanos, puesto que ellos son los verdaderos evaluadores de la actividad asistencial y de la calidad del producto ofrecido.

El modelo de gestión asistencial del Servicio de Oftalmología del Hospital La Moraleja de Sanitas (1), tiene como soporte primordial el principio de accesibilidad total. Nuestro objetivo es ofrecer al paciente nuevas alternativas a los servicios asistenciales tradicionales (cita previa, urgencias), que se adapten a las necesidades particulares de todos y cada uno de ellos, dentro del actual marco sanitario de creciente demanda de servicios y que mejoren los indicadores de calidad percibida y satisfacción global. En definitiva, se trata de organizar los recursos disponibles al objeto de facilitar el acceso inmediato a la consulta con una asistencia exacta y puntual proporcionando un «producto» acorde con las necesidades y expectativas de los pacientes.

\section{DESCRIPCIÓN DEL MODELO DE GESTIÓN DE ACCESIBILIDAD TOTAL}

El modelo que se describe a continuación se encuentra operacional desde abril de 2007. La consulta de oftalmología se desarrolla en $1 .{ }^{\text {er }}$ lugar con los pacientes previamente citados, con intervalos de quince minutos, durante doce horas todos los días, de lunes a viernes. La media de agendas simultáneas/día es de tres en horario matinal y cuatro vespertinas. A ello hay que añadir una agenda programada los sábados por la mañana que lleva a cabo el oftalmólogo de guardia.

Durante el horario de consulta se realizan las exploraciones complementarias diagnósticas o terapéuticas necesarias salvo las campimetrías y angiografías fluoresceínicas (AFG), las cuales, por su mayor duración, disponen de agendas diferenciadas. Las restantes pruebas oftalmológicas se integran y ejecutan dentro del propio acto de consulta, con lo que podemos hablar propiamente de «consultas de alta resolución». Por otro lado se atienden todas las urgencias remitidas desde la puerta, las interconsultas de pacientes hospitalizados por otros especialistas y, finalmente, los chequeos de salud (7-8 diarios).

$\mathrm{Su}$ organización interna se estructura en secciones que corresponden a las diferentes subespecialidades (polo anterior, retina, glaucoma, oftalmología pediátrica y estrabismo, etc.), circunstancia necesaria y favorecida por el progresivo avance en conocimientos y tecnologías a nivel de todas las especialidades médicas, y que constituye la mejor forma de proporcionar una asistencia altamente especializada, en lo que a calidad científico-técnica y competitividad se refiere. Sin embargo y al objeto de no originar compartimentos estanco, esta ineludible organización subespecializada no se distribuye en agendas diferenciadas, es decir, es únicamente funcional pero no orgánica para evitar las consecuencias bien conocidas de los modelos organizativos constituidos en compartimentos estanco de escasa fluidez: demoras asistenciales y listas de espera.

La divergencia estriba en que todos los pacientes pueden citarse en las agendas de cualquier oftalmó-

\footnotetext{
${ }^{1}$ Doctor en Medicina. Ex-Director Médico y Gerente del Hospital Universitario de La Princesa. Madrid.

E-mail: oftalmologiahlm@sanitas.es
} 
logo, con la única excepción diferencial pero no rígida, entre oftalmología infantil o del adulto. Serán posteriormente los propios facultativos los que irán canalizando a los pacientes en las sucesivas revisiones y en función de su patología, al oftalmólogo subespecializado conveniente. En este sentido la propia práctica clínica justifica una organización más funcional y efectiva que el modelo de agendas subespecializadas desde la perspectiva de la prevención de listas de espera.

La conjunción de un intervalo medio de citas fijado en 15 minutos, unido a la presencia constante de 3 ó 4 oftalmólogos en la consulta, nos permite en la mayoría de las ocasiones no sólo absorber con fluidez toda la demanda, sino también la posibilidad de dar un salto cualitativo ofreciendo cobertura inmediata sin cita previa a todos aquellos pacientes que lo soliciten, ya sea en el propio control de citaciones o externamente a través del «Call-Center». La clave radica en el intervalo medio y en el número de oftalmólogos concurrentes, de forma que puedan proporcionarse las condiciones apropiadas y el ambiente necesario para que éstos puedan desempeñar su responsabilidad de un modo sereno y satisfactorio a la vez que constante, en unas condiciones óptimas que invaliden la percepción de angustia o inquietud.

Con este escenario puede resultar menos complicado alcanzar el segundo objetivo fundamental anteriormente mencionado: la puntualidad (en el paciente previamente citado) y la asistencia inmediata (en el paciente que acude sin cita previa); y lo que aun podría resultar más trascendente desde la perspectiva de la calidad percibida y sin precedentes organizativos — no así en muchas otras consultas y centros, en períodos y circunstancias puntuales- la asistencia anticipada a la mayoría de los pacientes que así lo posibilitan por llegar con antelación.

En conclusión, el modelo de gestión de accesibilidad total implica una rigurosa y estricta organización que proporcione una oferta siempre superior a la demanda, un alto nivel de preparación y formación, conocimientos y experiencia de los facultativos y del resto del personal sanitario y auxiliar, así como ciertas disposiciones que deberán estar presentes entre los miembros de la organización, las más importantes la generosidad y entereza para obtener beneficios no a corto plazo. Bajo este enfoque, algunas opiniones autorizadas podrían discutir el modelo por el hecho de sacrificar una mayor productividad cuantitativa, en beneficio de priorizar unas condiciones laborales holgadamente favora- bles para los profesionales implicados. Sin embargo, y en materia de asistencia sanitaria, donde el producto que se oferta es salud, organizaciones que fomenten y potencien mayor producción y rentabilidad inmediatas, además de convertirse con el tiempo en inflexibles, muy probablemente generen tensiones entre los profesionales con las consiguientes pérdidas en calidad asistencial y competencia a medio y largo plazo.

Un efecto cuantificable de este modelo de gestión es el relacionado con la observación de la ocupación de las salas de espera, comparativamente con las de otros servicios adyacentes: Oftalmología, situándose en 2006-2007 entre los cuatro primeros servicios del hospital en volumen de consultas junto a Obstetricia-Ginecología, Traumatología y ORL, dispone de una sala de espera que usualmente muestra índices de ocupación medio-bajos, lo cual puede interpretarse por un flujo de entrada y salida de pacientes acorde con la demanda generada, y por una asistencia que se lleva a cabo con puntualidad. Podríamos formular que el índice de ocupación de una sala de espera es inversamente proporcional a la capacidad del servicio para absorber su demanda. Mayores dificultades tendríamos para enjuiciar los beneficios de esta evidencia: aparentaría ser ventajoso en lo que respecta a liberar espacios, reducir el murmullo de la sala y proporcionar mayor comodidad; por el contrario, podría ser negativa la percepción de un servicio de peor calidad dada la eventualidad de una sala de espera con su aforo a un tercio o media entrada. Probablemente el término medio sea lo más eficaz.

El modelo asistencial de accesibilidad total se complementa con las consultas de los sábados. Éstas funcionan igual que las de cita previa, siendo su diseño y ejecución resultado de dos condicionantes manifiestos: primero, una parte considerable de los pacientes muestran dificultades para asistir al médico durante su jornada ordinaria de trabajo, encontrando en los sábados por la mañana el momento ideal para efectuar dicha consulta; la misma consideración puede aplicarse a niños y estudiantes durante su jornada escolar semanal. Segundo, la presencia del oftalmólogo en el hospital los sábados por la mañana garantiza la cobertura de las urgencias, ya que éste se encuentra físicamente y no de forma localizada como el resto del sábado, domingos y festivos; por otra parte, permite organizar otras actividades (p.e. chequeos médicos) con idénticos recursos. 


\section{PILARES DEL MODELO DE GESTIÓN ASISTENCIAL DE ACCESIBILIDAD TOTAL}

1. Gestión de consultas ordinarias con cita previa

a) Acceso inmediato - ausencia de listas de espera

b) Atención inmediata - puntualidad o anticipación

c) Consultas de alta resolución

d) Consultas simultáneas - «trabajo en equipo»

e) Intervalos de citación de 15 minutos

f) Formación, experiencia y subespecialización

g) Dotación de tecnologías emergentes

h) Organización funcional

2. Gestión de consultas sin cita

a) Acceso y atención inmediatos

b) Integración en agendas ordinarias

c) Oferta superior a demanda conocida

3. Asistencia complementaria

a) Urgencias en horario de consulta

b) Interconsultas de pacientes ingresados

4. Gestión de consultas de sábados

a) Ofrece alternativas horarias al paciente

b) Cobertura especializada de urgencias

\section{DECÁLOGO DE IMPLANTACIÓN DEL MODELO DE ACCESIBILIDAD TOTAL}

1. Alto grado de compromiso de los miembros de la organización con los principios de filosofía asistencial del servicio.
2. Elevado nivel de formación, cualificación y experiencia profesional. Desarrollo de la subespecialización.

3. Intervalos de citación (15 min.) adaptados a una atención sanitaria optimizada, serena y constante. Condiciones óptimas de trabajo.

4. Programación de al menos tres consultas especializadas simultáneas.

5. Oferta superior a la demanda asistencial conocida.

6. Dotación de los medios técnicos necesarios para la realización de pruebas oftalmológicas.

7. Incentivación y motivación de los profesionales: Retribución por acto médico, productividad, disponibilidad de tecnologías emergentes, formación médica continuada, docencia e investigación.

8. Implicación de los profesionales en la gestión del servicio: Dirección participativa por objetivos (DPO). Transparencia en la gestión.

9. Organización, planificación, evaluación y control liderados por el jefe de servicio. Autonomía de gestión.

10. Estrecha colaboración con los órganos de gobierno y objetivos generales del hospital. Planes estratégicos del hospital y servicio.

\section{BIBLIOGRAFÍA}

1. Gegúndez JA. Proyecto Técnico de Gestión. Madrid: Hospital La Moraleja, Servicio de Oftalmología; 2003 Dic. 123 pp. 